\title{
Common Core Offers the Opportunity to Reorder Math Topics to Better Emphasize Their Connections and Applications
}

\author{
Susan Schwartz Wildstrom
}

I am moved to respond to Sol Garfunkel's "Opinion" article. ${ }^{1}$ I am a long-time high school mathematics teacher in a public school. I started teaching around the time of SMSG and have been in the trenches throughout several of the math wars. I know Dr. Garfunkel's fine work in creating interesting modeling projects and his outspoken opinion that using technology to solve problems that apply the mathematics we are teaching will better concretize students' understanding of the underlying mathematics. It sounds like a fine idea, but the reality is often very different.

Our problems in teaching mathematics begin in elementary school. Sadly, many teachers working with our children at the start of their mathematical

Susan Schwartz Wildstrom is a mathematics teacher at Walt Whitman High School in Montgomery County, Maryland. Her email address is susan@ wi 1dstrom. com.

Members of the Editorial Board for Doceamus are: David Bressoud, Roger Howe, Karen King, William McCallum, and Mark Saul.

${ }^{1}$ Sol GARfunKel, "What's a Math Educator to Do?", Notices Amer. Math. Soc. 59 (2012), 909.

DOI: http://dx.doi.org/10.1090/noti984 journeys are not themselves comfortable with the mathematics they are trying to teach. They often only know one way to teach an idea and they may not fully understand how that method works and why it gives the right answers. Such a teacher confronted with an alternate creative method (perhaps suggested by a clever child or a seasoned colleague) may reject the alternative rather than trying to see how and why two methods produce the same result. Beyond stifling the creativity of students and discouraging them from trying to see how the mathematics works, such an approach is not fertile ground for applications and modeling projects in which creative exploration and possibly unorthodox methods are encouraged as a means of truly understanding what is happening. Teachers who lack confidence in their own understanding of the ideas may not want to include these sorts of activities in their classrooms.

As we implement the Common Core State Standards (CCSS) with their emphasis on fewer concepts more deeply taught, we need to ensure that our elementary school teachers are themselves learning and understanding multiple ways to solve individual problems and that they get their hands dirty in the applications and models that can be used alongside the traditional solution methods. 
Perhaps future preservice math classes will need to include labs that develop concepts from basic principles and contain examples of applications and modeling.

Moving forward, we see that in many algebra classes real-world collections of data are incorporated into some of the problems we use in our classes. A popular exercise involves giving students a collection of ordered pairs from experimental data that represents an unknown but linear relationship. The students' task is to find the best fit line. In many classes this is done by having the students enter the list of ordered pairs into calculators, after which they push a sequence of buttons on the calculator which then produces a linear equation for the line of best fit. They are later similarly taught to use the built-in functions for quadratic, exponential, and logarithmic regressions as well. From my observation of how this is done and taught, students don't really get an understanding of the underlying mathematical principles; they are only interested in getting the right answer, and they just want to be sure that they push the right buttons at the right times. Learning the algorithmic technological approach to solving a problem is a far cry from using modeling for an interesting application to understand mathematics. I am aware that a student in her first algebra class is probably not going to be able to understand the underlying mathematics of the least squares model, but I also do not believe that this is a particularly effective application or modeling exercise, rather something that can be used to answer a question that will appear on a state assessment.

A modeling project or a well-crafted application of a mathematical concept can reinforce understanding the underlying mathematics if it has been clearly taught and the student understands how and why it works. A poorly understood concept will not be clarified by a complex application problem, no matter how interesting or engaging the setting may be.

Dr. Garfunkel fears that CCSS might eliminate a place for modeling and applications. My opinion is completely opposite. Fewer concepts more thoroughly taught at each level would let teachers spend more time on each idea, and that could leave more time to bring in the interesting applications, connections, and extensions that are at the heart of Garfunkel's philosophy. Curriculum implementing CCSS is now being written. What a wonderful opportunity to add in strong, well-crafted examples that will reinforce what children are learning. (A trivial elementary school example might be to bring in population, income, and similar numbers to show students what large numbers look like. This would allow discussion of how to compare their sizes and estimate how large such numbers are without getting lost in the weeds of the values in the units and tens places, emphasis on place value, and how more and more digits to the left indicate larger and larger amounts, etc.)

Another of my own pet peeves (and something that CCSS could endeavor to fix) is the way we talk about making connections among mathematical concepts but in reality often teach them as though each new concept were an idea unrelated to anything students have seen before. A personal favorite is the way many algebra textbooks teach factoring of quadratic polynomials, completing the square to solve quadratic equations, the quadratic formula for solving equations, and graphing of quadratics in four different places in the course. To make matters worse, many teachers do not (when they have already taught one of these topics and are teaching another of them) take the time to connect the material to a topic already learned. So students come to think of mathematics as a set of topics, each one new and unrelated to anything they have done before. Go back to square one and start all over. I wonder how much teaching time is lost in setting the stage for new topics as if they had no connection to something students already know. Anytime we make a clear connection to ideas already learned, we give students a starting point, a set of ideas on which they can build, and a way of seeing how things connect. Using what you already know to solve a more complex problem prepares students for modeling and applications.

Over the course of the forty-plus years that I have been teaching, I have seen many "new" approaches intended to improve the teaching of math. Each probably contributed in some small way to the way I actually teach math in my classroom. But I understand and like math, so I can see how new tactics fit into what I am doing. Someone who is not confident might be unable to adapt and include new ideas in how they teach.

I believe that children begin their schooling excited about learning and eager to engage in the process. Keeping that excitement alive is key to helping students learn and understand mathematics. I hope that, as CCSS is implemented, a high priority will be adapting the way we prepare the teachers who will bring these implementations to the children in our classrooms so that these students can learn to apply the mathematics they are learning to their lives in meaningful ways. 